\title{
Probable Longer Incubation Period for Elderly COVID-19 Cases: Analysis of 180 Contact Tracing Data in Hubei Province, China
}

This article was published in the following Dove Press journal: Risk Management and Healthcare Policy

\author{
Jingyi Dai ${ }^{\prime}$ \\ Lin Yang ${ }^{2}$ \\ Jun Zhao $\mathbb{D}^{2}$
}

'Department of Infectious Diseases, The Third People's Hospital of Kunming City, Kunming, Yunnan Province, People's Republic of China; ${ }^{2}$ School of Public Health and Management, Hubei University of Medicine, Shiyan, Hubei Province, People's Republic of China
Correspondence: Jun Zhao

School of Public Health and Management, Hubei University of Medicine, 30 South Renmin Road, Shiyan, Hubei 442000,

People's Republic of China

Email stzhao@I63.com
Background: Factors associated with the incubation period of COVID-19 are not fully known. The aim of this study was to estimate the incubation period of COVID-19 using epidemiological contact tracing data, and to explore whether there were different incubation periods among different age grloups.

Methods: We collected contact tracing data in a municipality in Hubei province during the full outbreak period of COVID-19. The exposure periods were inferred from the history of travel in Wuhan and/or history of exposure to confirmed cases. The incubation periods were estimated using parametric accelerated failure time models accounting for interval censoring of exposures.

Results: The incubation period of COVID-19 follows a Weibull distribution and has a median of 5.8 days with a bootstrap 95\% CI: 5.4-6.7 days. Of the symptomatic cases, 95\% showed symptoms by 14.3 days (95\% CI: 13.0-15.7), and 99\% showed symptoms by 18.7 days (95\% CI: 16.7-20.9). The incubation periods were not found significantly different between male and female. Elderly cases had significant longer incubation periods than young age cases (HR 1.49 with 95\% CI: 1.09-2.05). The median incubation period was estimated at 4.0 days (95\% CI: 3.5-4.4) for cases aged under 30, 5.8 days (95\% CI: 5.6-6.0) for cases aged between 30 and 59, and 7.7 days (95\% CI: 6.9-8.4) for cases aged greater than or equal to 60 .

Conclusion: The current practice of a 14-day quarantine period in many regions is reasonable for any age. Older people infected with SARS-CoV2 have longer incubation period than that of younger people. Thus, more attention should be paid to asymptomatic elderly people who had a history of exposure.

Keywords: infectious disease, coronavirus, incubation period, epidemiology

\section{Introduction}

A novel coronavirus disease, designated as COVID-19, has become a pandemic worldwide. As of March 29, 2020, there were 634,835 confirmed COVID-19 cases and 29,957 deaths reported worldwide. ${ }^{1}$ However, we still have only a limited understanding about many aspects of our common enemy, such as the transmission dynamics. $^{2}$ The incubation period is defined as the time from infection to symptom onset. It is a key indicator that helps to depict the transmission dynamics of infectious diseases. ${ }^{3}$ It can also help to determine the quarantine period for suspicious cases. ${ }^{4,5}$

Thus far, several studies have estimated the incubation period of COVID-19. The reported mean incubation period for COVID-19 varied from 4 days to 10.9 
days. ${ }^{6-9}$ However, the above-mentioned studies have been limited by their small sample sizes and/or the fact that they were based on publicly reported data. As a result, sampling bias and selection bias may have been introduced into the estimates. ${ }^{10}$ Moreover, factors associated with the incubation period of COVID-19 are not fully known.

To obtain reliable estimates of the incubation period for COVID-19, we collected data in a municipality in Hubei province during a full outbreak period. Our aim was to further assess the incubation period of COVID-19, and to explore whether there were different incubation periods among different age groups.

\section{Methods}

\section{Study Setting Background}

On 20 January 2020, the Shiyan Center for Disease Control and Prevention in Hubei province identified the first COVID-19 case and began active surveillance of people returning from Wuhan and its surrounding areas in Hubei province. After Wuhan announced its lockdown on 23 January 2020, Shiyan shut down its traffic two days later, and then closed communities and villages the following day, on 26 January 2020 . The surveillance program was then expanded to people who had recently returned from Wuhan regardless of symptoms; patients in hospitals or clinics; and individuals detected by fever screening in communities. Epidemiological surveys were conducted for each confirmed case, using a standard format.

\section{Data Collection}

We collected data for cases with confirmed COVID-19 in Shiyan based on the epidemiological survey reports. Information collected included each case's sex, age, source of infection (imported from Wuhan, or locally infected), date of exposure (entry into and exit from Wuhan, or the earliest and latest date of close contact with a Wuhanimported/locally infected case), date of symptom onset, and date of diagnosis.

The symptom onset of COVID-19 patients was defined as one or more of the following symptoms: cough, dyspnea, fever, chest congestion, muscle soreness, chills, fatigue, and diarrhea. To estimate the incubation period for COVID-19, the exposure periods were inferred from the history of travel in Wuhan and/or history of exposure to confirmed cases, as follows: (i) If a patient had a history of travel in Wuhan two weeks before symptom onset and stayed no more than one week in Wuhan, the left and right endpoints of the windows of possible date of infection were set as the date of entry into and exit from Wuhan, respectively; (ii) If a patient did not have a history of travel to Wuhan two weeks before symptom onset and had a well-defined contact period with a confirmed case, the left and right endpoints of the windows of possible date of infection were set as the initial contact date and the last contact date, respectively; and (iii) If a patient had a history of staying in Wuhan for more than one week, or if there was no clear contact information about a confirmed case, or if the contact period with a confirmed case was not clear or was longer than one week, the exposed period was not recorded.

\section{Statistical Analysis}

Frequency counts and percentages were used in the descriptive analysis. All of the analyses were performed using $\mathrm{R}$ software, version 3.6.1. ${ }^{11}$

For each case, the date of infection was between two possible dates, while the date of symptom onset was definite. Such data are called single interval-censored data. ${ }^{12}$ We estimated incubation period using the algorithm described by Reich et al implemented in the coarseDataTools R package. ${ }^{13}$ The incubation period distributions were estimated using three commonly used distributions (Weibull, gamma, and lognormal). ${ }^{14-16}$ We chose the best fit model by comparing the $\log$-likelihood values of the three distributions. We then estimated the means and specific quantiles (5th, 50th, 95th, and 99th percentiles) for each distribution. To compare the incubation periods among cases with different age groups, we fitted the model which best fits the data and plotted the distribution for each age group. Finally, we applied a multivariate regression using the best fit model to examine the difference in incubation periods for different age groups.

\section{Ethics Statement}

This study was approved by the Research Ethics Committee of Hubei University of Medicine to access the database and analyze the data. Analytic datasets were constructed in an anonymized fashion. The names and identification numbers of all patients were encrypted before use to ensure confidentiality. Approval to conduct the study was done in compliance with the Declaration of Helsinki.

\section{Results}

\section{Patients' Characteristics}

Between 20 January 2020 and 29 February 2020, 672 COVID-19 cases were detected and diagnosed in Shiyan, 
Hubei province. We identified 180 cases with a welldefined period of exposure and date of symptom onset. Of the 180 cases, the mean age was 46.1 years with a standard deviation of 15.3 years; $83(46.1 \%)$ were female and $97(53.9 \%)$ were male; 57 (31.7\%) had a history of staying in Wuhan no more than one week prior to onset or diagnosis, and $123(68.3 \%)$ were locally infected. Detailed exposure to symptom onset timeline for these 180 cases is presented in Figure 1. The length of exposure intervals ranged from 0 to 7 days with a mean of 2.3 days and a standard deviation of 2.4 days.

\section{Incubation Period Distribution}

Table 1 presents the estimated incubation periods for COVID-19 using three different parametric models. Of the three fitted distributions, the Weibull distribution has the largest log-likelihood, thus provides the best fit to the data. From the Weibull model, the estimated incubation period of all COVID-19 cases has a median of 5.8 days with a bootstrap 95\% CI: 5.2-6.4 days. Among all the symptomatic cases, $5 \%$ showed symptoms by 1.2 days (95\% CI: $0.9-1.5)$ after infection, 95\% showed symptoms

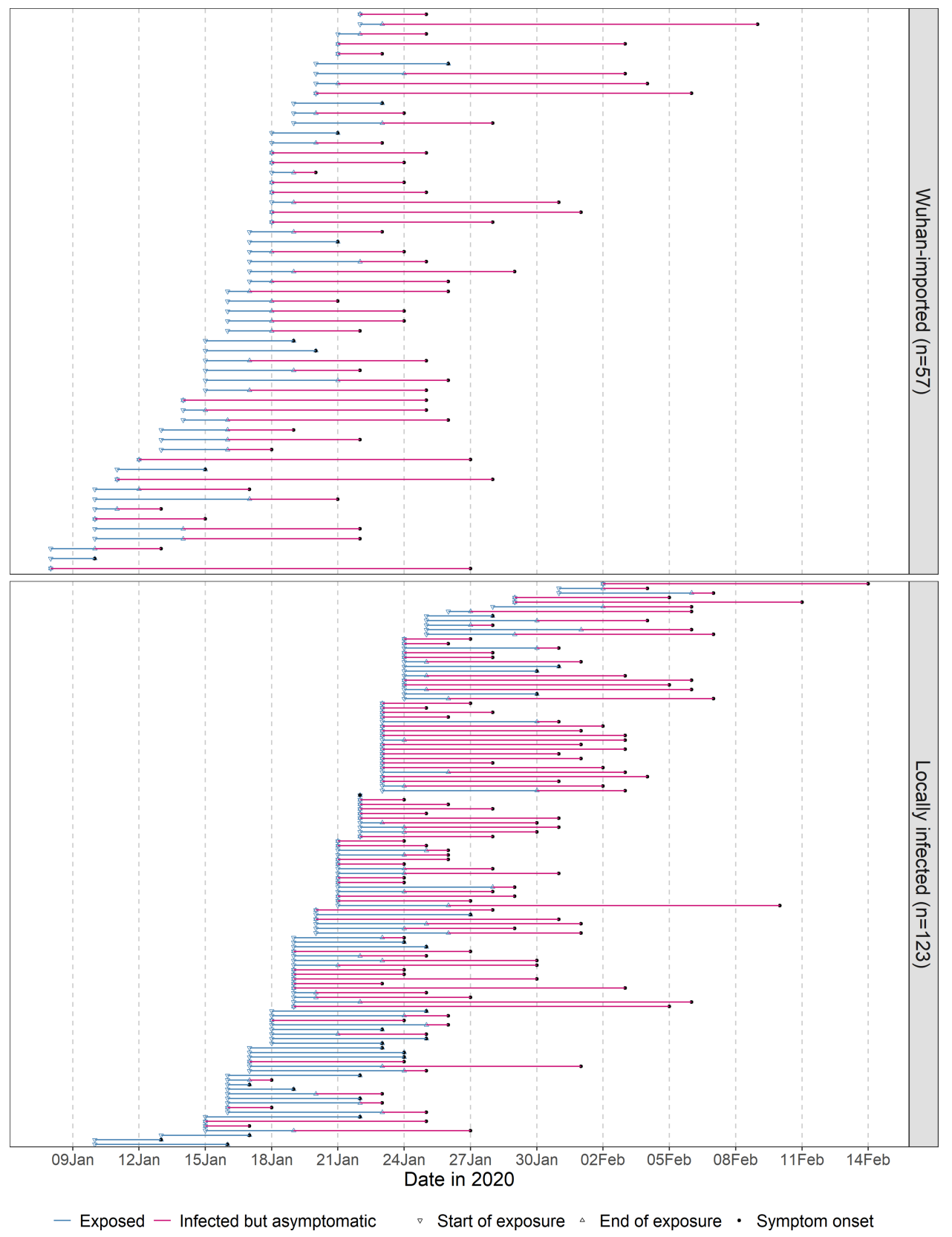

Figure I Exposure to symptom onset timeline for Wuhan-imported (upper panel) or locally infected (lower panel) COVID-19 cases sorted by earliest exposure date. 
Table I Estimated Incubation Period for COVID-19 Cases Using Different Parametric Models

\begin{tabular}{|c|c|c|c|c|c|c|c|c|}
\hline Distribution & $\begin{array}{l}\text { Log- } \\
\text { Likelihood* }\end{array}$ & Parameter I & Parameter 2 & Mean & $\begin{array}{l}5^{\text {th }} \\
\text { Percentile }\end{array}$ & $\begin{array}{l}50^{\text {th }} \\
\text { Percentile }\end{array}$ & $\begin{array}{l}95^{\text {th }} \\
\text { Percentile }\end{array}$ & $99^{\text {th }}$ Percentile \\
\hline Weibull & -449.5 & $1.6(1.4-1.9)$ & $7.3(6.6-7.9)$ & $6.5(5.9-7.1)$ & $1.2(0.9-1.5)$ & $5.8(5.2-6.4)$ & $14.3(13.0-15.7)$ & $18.7(16.7-20.9)$ \\
\hline gamma & -452.7 & $2.1(1.5-3.0)$ & $3.0(2.2-4.2)$ & $6.5(5.9-7.1)$ & $1.3(0.7-1.8)$ & $5.5(4.8-6.2)$ & I5.I (13.4-16.9) & $21.0(18.1-24.1)$ \\
\hline Log-normal & -479.3 & $1.6(1.5-1.8)$ & $0.9(0.6-1.2)$ & $7.2(6.2-8.9)$ & $1.2(0.6-2.1)$ & $5.0(4.3-5.9)$ & $20.5(14.7-29.5)$ & $36.6(22.2-58.8)$ \\
\hline
\end{tabular}

Note: *Larger value of log-likelihood indicates a better fit.

Table 2 Parameter Estimates and 95\% Confidence Intervals of Weibull Distributions Used to Estimate the Incubation Period for COVID-19 Cases

\begin{tabular}{|c|c|c|c|c|}
\hline \multirow[t]{2}{*}{ Parameter } & \multirow[t]{2}{*}{ All $(n=180)$} & \multicolumn{3}{|l|}{ Age (Years) } \\
\hline & & $<30(n=27)$ & $30-59(n=123)$ & $\geq 60(n=30)$ \\
\hline Shape & $1.6(1.4-1.9)$ & $1.3(0.8-1.8)$ & $1.6(1.4-1.9)$ & $2.4(1.6-3.2)$ \\
\hline Scale & $6.5(5.9-7.1)$ & $5.3(3.5-7.1)$ & $7.3(6.4-8.2)$ & $8.9(7.4-10.4)$ \\
\hline 5th percentile & $1.2(0.9-1.5)$ & $0.5(0.2-0.9)$ & $1.1(1.0-1.3)$ & $2.6(2.0-3.2)$ \\
\hline 50th percentile & $5.8(5.2-6.4)$ & $4.0(3.5-4.4)$ & $5.8(5.6-6.0)$ & $7.7(6.9-8.4)$ \\
\hline 95th percentile & $14.3(13.0-15.7)$ & $12.3(\mid 1.7-12.8)$ & $14.4(14.2-14.7)$ & I4.I (13.2-15.0) \\
\hline 99th percentile & I8.7 (16.7-20.9) & $17.1(16.5-17.6)$ & $18.9(18.6-19.2)$ & $16.9(15.9-17.8)$ \\
\hline
\end{tabular}

by 14.3 days ( $95 \%$ CI: $13.0-15.7$ ), and $99 \%$ showed symptoms by 18.7 days (95\% CI: 16.7-20.9) (Table 2).

The parameters and specific quantiles of Weibull distributions fitted to incubation periods for different age groups are shown in Table 2. The shape and scale parameters of the corresponding distributions increase as the age group changes from the youngest to the eldest. Figure 2 shows the corresponding probability density distributions and cumulative density distributions of incubation periods for the three age groups. The incubation periods have an

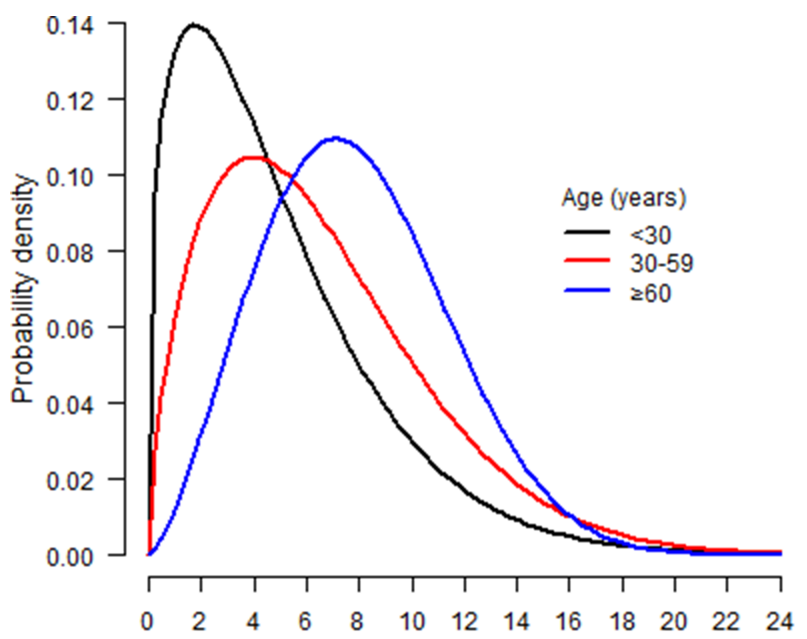

Time from infection to symptom onset (days) increasing trend from the youngest to the eldest cases. The median incubation period was estimated at 4.0 days (95\% CI: 3.5-4.4) for cases aged under 30, 5.8 days (95\% CI: 5.6-6.0) for cases aged between 30 and 59, and 7.7 days (95\% CI: 6.9-8.4) for cases aged greater than or equal to 60 . The three distribution curves are almost superimposed in the right tails after 16 days. Of those symptomatic cases, $95 \%$ cases aged less than 30 showed symptoms within 12.3 days (95\% CI: 11.7-12.8) after infection, while 14.4 days (95\% CI: 14.2-14.7) for cases aged between 30 and 59, and

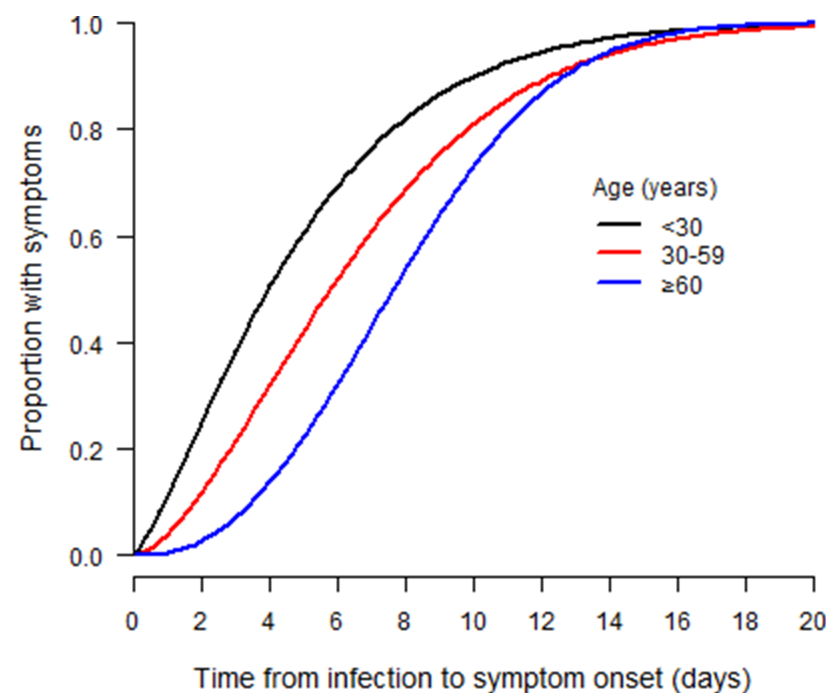

Figure 2 Probability density function (left panel) and cumulative distribution function (right panel) of the Weibull distribution used to estimate the distribution of incubation periods by age group for 180 COVID-19 cases in Hubei, China. 
Table 3 Association Between Incubation Periods and Characteristics of COVID-19 Cases in Multivariate Weibull Regression Model

\begin{tabular}{|l|l|l|l|}
\hline Variables & HR (95\% CI) & $\begin{array}{l}\text { P value } \\
\text { (Wald Test) }\end{array}$ & $\begin{array}{l}\text { P value } \\
\text { (LRT) }\end{array}$ \\
\hline $\begin{array}{c}\text { Sex } \\
\text { Female } \\
\text { Male }\end{array}$ & $\begin{array}{l}\text { reference } \\
\text { I.06 }(0.88,1.27)\end{array}$ & 0.549 & 0.549 \\
\hline $\begin{array}{c}\text { Age (years) } \\
<30 \\
30-59 \\
\geq 60\end{array}$ & $\begin{array}{l}\text { reference } \\
1.27(0.99,1.64)\end{array}$ & 0.065 & 0.048 \\
\hline $\begin{array}{c}\text { Source of infection } \\
\text { Wuhan-imported } \\
\text { Locally infected }\end{array}$ & $\begin{array}{l}0.49(1.09,2.05) \\
\text { reference }(0.68,1.00)\end{array}$ & 0.013 & \\
\hline
\end{tabular}

Abbreviations: $\mathrm{HR}$, hazard ratio; $\mathrm{Cl}$, confidence interval; LRT, log-likelihood ratio test.

14.1 days (95\% CI: 13.2-15.0) for cases aged greater than or equal to 60 .

In the multivariate Weibull regression model (Table 3), sex and source of infection were not found to be significantly associated with the incubation period. The incubation periods for elderly cases and middle-age cases were longer than those for young cases, though only the elderly showed a significant longer incubation period than the young cases $(\mathrm{P}=0.013)$. After adjusting for sex and source of infection, the incubation period for cases above 60 years was 1.5 times (95\% CI: 1.09-2.05) longer than that of cases aged less than 30 on average.

\section{Discussion}

Using contact tracing data accounting for interval censoring of exposures, our estimate of incubation period for COVID-19 follows a Weibull distribution with a median of 5.8 days. The incubation period for elder cases is significantly longer than that for younger cases. Our estimated median incubation period is longer than the values from two studies conducted during the early outbreak of COVID-19, which were 5.2 days $^{17}$ and 4.8 days, ${ }^{18}$ respectively. These two studies set the date of leaving Wuhan as the exposure time. However, infectees may have been exposed several days prior to leaving Wuhan. This might result in an underestimate of the incubation period.

Time of infection is critical in estimating the incubation period. The Dongfeng Motor Corporation, founded in Shiyan in 1969, is now headquartered in Wuhan. However, many of its manufacturing plants are still in Shiyan. As a result, there is more frequent population mobility between these two cities than other cities in the province. In the first week after Wuhan lockdown, Shiyan was one of the four cities with the fastest growth in the number of COVID-19 cases in the province, although it is geographically farthest from Wuhan in the province. Of all the cases imported from Wuhan, about one-third were related to the Dongfeng Motor Corporation. This facilitated our ability to estimate the time of infection in cases with a history of short stay in Wuhan before symptom onset. Besides, because of the lockdown of the communities in the city, most transmissions of COVID-19 occurred within families or neighborhoods. Therefore, for those had close contacts with Wuhanimported cases, we could infer source of infection with high accuracy. As a result, we can obtain the time interval of infection from the earliest and last contact time. For these reasons, our estimate of the incubation period should be more justifiable than those in previously published studies.

Although the incubation period distributions of different age groups are significantly different, the distribution curves are almost superimposed in the right tails. Of symptomatic cases in any age group, about 95\% will show symptoms within 14 days. This supports the currently practiced length of quarantine in many countries. However, considering that nearly $5 \%$ of patients still require an incubation period of more than 14 days, a 1-5-day extension of the quarantine period may be necessary.

Our results suggest that older people infected with SARS-CoV-2 have longer incubation period than that of younger people. The more responsive the host's immune system is to a respiratory virus, the shorter the incubation period. ${ }^{19}$ Elderly people display a marked increase in the proportion of highly differentiated effector and memory $\mathrm{T}$ cells due to a lifetime of exposure to a variety of pathogens. ${ }^{20}$ A significant proportion of $\mathrm{T}$ cells of elderly loses the expression of costimulatory molecules, ${ }^{20-22}$ which may lead to delay in the transmission of defense information by the immune system. When the host's immune system is not yet activated, the virus can replicate in large amount in the incubation period. This might explain that the proportion of severe cases in elder age was significantly higher than in the young. ${ }^{23}$ The older has longer incubation period yet we do not know whether they shed the virus for a longer asymptomatic period than the young ones. This need to be proven by another epidemiological study.

The present study has several limitations. First, the data were extracted from epidemiological survey reports. 
Recall bias was inevitable, particularly for the key timing variables. Patients returned from Wuhan may have delayed reporting their symptoms to avoid discrimination. For these cases, we may overestimate the incubation periods. Second, we assigned a uniform probability distribution to the exposure interval for cases during their staying in Wuhan, and for cases during their contacting Wuhanimported cases. It is possible that the time of infection is more inclined to the left or right endpoint of the interval, depending on the transmission of SARS-CoV2. We limited the length of intervals to within seven days (nearly $80 \%$ were within four days) to minimize this potential bias. In addition, we exclude cases who travelled in Wuhan for more than one week to obtain more reliable exposure time periods. A large proportion of Wuhan residences were excluded. This leads to a bias against identifying longer incubation periods in the Wuhan-arrivals. Third, we only had information on confirmed cases and may have missed some cases with very mild symptoms. In view of the large sample size, it is unlikely that these biases could have influenced the estimation very much.

In conclusion, our results contribute to a better understanding of COVID-19 and provide useful parameters for modeling the dynamics of disease transmission. The current practice of a 14-day quarantine period in many regions is reasonable for any age. However, more attention should be paid to asymptomatic elderly people who had a history of exposure.

\section{Acknowledgments}

We thank all the staffs and health workers who did the contact tracing and collected the data. This work was supported by the Special Emergency Research Project on COVID-19 at Hubei University of Medicine (Grant No. 2020XGFYZR06), and Shiyan Emergency Scientific Research Project of COVID-19 Prevention and Control Technology (Grant No. 20Y02).

\section{Disclosure}

The authors report no conflicts of interest in this work.

\section{References}

1. WHO. Coronavirus disease 2019 (COVID-19) Situation Report-80; 2020. Available from: https://www.who.int/emergencies/diseases/ novel-coronavirus-2019/situation-reports. Accessed July 30, 2020.

2. Cowling B, Leung G. Epidemiological research priorities for public health control of the ongoing global novel coronavirus (2019-nCoV) outbreak. Eurosurveillance. 2020;25(6):pii=2000110. doi:10.2807/ 1560-7917.ES.2020.25.6.2000110
3. Cohen JE. Infectious diseases of humans: dynamics and control. JAMA. 1992;268(23):3381. doi:10.1001/jama.1992.03490230111047

4. Lessler J, Reich NG, Cummings DAT. Outbreak of 2009 Pandemic Influenza A (H1N1) at a New York City School. $N$ Eng $J$ Med. 2009;361(27):2628-2636. doi:10.1056/NEJMoa0906089

5. Nishiura H. Determination of the appropriate quarantine period following smallpox exposure: an objective approach using the incubation period distribution. Int J hygiene Environ health. 2020;212(1):97-104.

6. Guan WJ, Ni ZY, Hu Y, et al. Clinical Characteristics of Coronavirus Disease 2019 in China. $N$ Engl J Med. 2020;382(18):1708-1720. doi:10.1056/NEJMoa2002032

7. Linton NM, Kobayashi T, Yang Y, et al. Incubation period and other epidemiological characteristics of 2019 novel coronavirus infections with right truncation: a statistical analysis of publicly available case data. J Clin Med. 2020;9(2):538. doi:10.3390/jcm9020538

8. You C, Deng Y, Hu W, et al. Estimation of the time-varying reproduction number of COVID-19 Outbreak in China. medRxiv Preprint. 2020:113555.

9. Ke Men XW. Estimate the incubation period of coronavirus 2019 (COVID-19). medRxiv Preprint. 2020.

10. Lauer SA, Grantz KH, Bi Q, et al. The incubation period of coronavirus disease 2019 (COVID-19) from publicly reported confirmed cases: estimation and application. Ann Intern Med. 2020;M20-0504.

11. Core Team R (2019). R: A language and environment for statistical computing. R Foundation for Statistical Computing, Vienna, Austria. Available from: https://www.R-project.org/. Accessed July 30, 2020.

12. Turnbull WB. Nonparametric estimation of a survivorship function with doubly censored data. Pub Am Statis Assoc. 1974;69 (345):169-173. doi:10.1080/01621459.1974.10480146

13. Reich NG, Lessler J, Cummings DA, Brookmeyer R. Estimating incubation period distributions with coarse data. Stat Med. 2009;28 (22):2769-2784. doi:10.1002/sim.3659

14. Backer JA, Klinkenberg D, Wallinga J. Incubation period of 2019 novel coronavirus $(2019-\mathrm{nCoV})$ infections among travellers from Wuhan, China, 20-28 January 2020. Euro Surveillance. 2020;25 (5):2000062.

15. Virlogeux V, Li M, Tsang TK, et al. Estimating the distribution of the incubation periods of human avian Influenza A(H7N9) Virus Infections. Am J Epidemiol. 2015;182(8):723-729. doi:10.1093/aje/kwv115

16. Rudolph KE, Lessler J, Moloney RM, Kmush B, Cummings DA. Incubation periods of mosquito-borne viral infections: a systematic review. Am J Trop Med Hyg. 2014;90(5):882-891. doi:10.4269/ ajtmh.13-0403

17. Li Q, Guan X, Wu P, et al. Early transmission dynamics in Wuhan, China, of novel coronavirus-infected pneumonia. $N$ Engl $\mathrm{J}$ Med. 2020;382(13):1199-1207. doi:10.1056/NEJMoa2001316

18. Liu T, Hu J, Xiao J, et al. Time-varying transmission dynamics of Novel Coronavirus Pneumonia in China. bioRxiv. 2020.

19. Hermesh T, Moltedo B, López CB, Moran TM. Buying time- the immune system determinants of the incubation period to respiratory viruses. Viruses. 2010;2(11):2541-2558. doi:10.3390/v2112541

20. Alonso-Arias R, Moro-Garcia MA, Lopez-Vazquez A, et al. NKG2D expression in CD4+ $\mathrm{T}$ lymphocytes as a marker of senescence in the aged immune system. Age. 2011;33(4):591-605. doi:10.1007/s11357010-9200-6

21. Fakhri H, Nelson LS, Carmen C, et al. Differential effect of cytomegalovirus infection with age on the expression of CD57, CD300a, and CD161 on T-cell subpopulations. Front Immunol. 2017;8:649. doi:10.3389/fimmu.2017.00649

22. Roux A, Mourin G, Larsen M, Fastenackels S, Appay V. Differential impact of age and cytomegalovirus infection on the $t$ cell compartment. Jl Immunol. 2013;191(3):1300-1306. doi:10.4049/ jimmunol.1202940

23. Verity R, Okell LC, Dorigatti I, et al. Estimates of the severity of coronavirus disease 2019: a model-based analysis. Lancet Infect Dis. 2020;20(6):669-677. doi:10.1016/S1473-3099(20)30243-7 


\section{Publish your work in this journal}

Risk Management and Healthcare Policy is an international, peerreviewed, open access journal focusing on all aspects of public health, policy, and preventative measures to promote good health and improve morbidity and mortality in the population. The journal welcomes submitted papers covering original research, basic science, clinical \& epidemiological studies, reviews and evaluations, guidelines, expert opinion and commentary, case reports and extended reports. The manuscript management system is completely online and includes a very quick and fair peer-review system, which is all easy to use. Visit http://www.dovepress.com/testimonials.php to read real quotes from published authors. 